\title{
Les préverbes macédoniens et leurs équivalents en français : étude contrastive des modes d'expression de l'aspect dans une perspective didactique
}

\author{
Jovan Kostov ${ }^{1}$ \\ ${ }^{1}$ EA 4514 - PLIDAM - Pluralité des langues et des identités : didactique, acquisition, médiation, INALCO, 2, rue de \\ Lille, 75007, Paris - France.
}

\begin{abstract}
Résumé. Dans cet article, nous allons effectuer une analyse contrastive des différents modes d'expression de l'aspect dans les langues slaves du Sud (notamment le macédonien) et en français. Notre analyse s'inscrit dans la continuité de la réflexion démarrée dans nos travaux de recherche sur le traitement automatique de la flexion verbale dans une perspective didactique. L'aspect étant une catégorie indéniablement polymorphe, nous souhaiterions apporter une nouvelle approche d'analyse de cette catégorie dans les langues romanes dans le but de guider l'apprenant slavophone à un apprentissage efficace des valeurs aspectuelles et de leurs réalisations hétérogènes d'un groupe de langues à un autre. Notre démarche se veut à la fois réflexive et émane de la linguistique appliquée à la didactique des langues et des cultures. Nous empruntons cette démarche au philosophe américain Donald Schönn en examinant les différentes études de l'aspect dans les langues slaves du Sud, afin d'inciter l'apprenant slavophone du français à réfléchir aux possibilités d'établir des passerelles de ses propres connaissances métalinguistiques de cette catégorie polymorphe, ce qui lui permettrait d'étudier efficacement ses réalisations dans un système linguistique différent.
\end{abstract}

\begin{abstract}
Macedonian verbal prefixes and their equivalents in French : contrastive study of the aspect from a didactic perspective. In this article, we will present a contrastive analysis of the different modes of expression of the aspect in South Slavic languages (eg Macedonian) and French. Our analysis continues the reflection started in our research on the automatic processing of verbal inflection in a didactic perspective. The aspect is undeniably a polymorphic category and we would like to bring a new analytical approach to this category in Romance languages in order to guide the slavophone learner to determine aspectual values and their heterogeneous realizations from one language to another. Our approach is reflective and arises from the applied linguistics and the didactics of languages and cultures. We base our study on the recomendations of the American philosopher Donald Schönn and our goal is to review the various studies of the aspect in the South Slavic languages, to encourage the slavophone learner of French to analyze the different modes of aspectual expression in order to build bridges between his own metalinguistic knowledge that might help him to study the aspectual mecanism of a different linguistic system.
\end{abstract}

\section{Introduction}

L'approche réflexive dans le processus d'apprentissage d'une langue seconde n'est pas une nouveauté en didactique. De nombreuses études attestent l'efficacité d'une mise en situation de réflexion de la part du sujet-apprenant d'abord sur le fonctionnement de sa propre langue, avant de décortiquer un fait donné d'une langue qu'il apprend. A titre d'exemple, nous allons souligner le travail de D. A. Schönn (Schönn, 1984) qui donne un cadre amplement développé pour ce que le praticien (dans notre cas l'enseignant d'une langue) devrait mobiliser dans le processus de mise en place d'une scénarisation. 
Cette approche suppose que l'enseignant, et dans notre cas l'enseignant d'une langue étrangère, instaure une dynamique interactive de l'apprentissage et mette en place des mécanismes de comparaison de la langue et de la culture apprises avec une langue et une culture dites "de référence » qui peuvent être celles dans lesquelles l'apprenant évolue au quotidien, ou encore sa langue et sa culture maternelles. En supposant que l'apprenant mobilise inévitablement ses connaissances du système linguistique qui lui sert de référence et établit des passerelles et des oppositions qui lui rendent l'apprentissage plus facile, l'enseignant adapte son discours, mais aussi tente d'entrer dans l'univers de l'apprenant pour découvrir la façon dont il va transmettre une compétence linguistique et culturelle. A travers cette comparaison, l'apprenant se rend compte de l'hétérogénéité des systèmes linguistiques et culturels, ce qui, dans la plupart des cas, lui sert de moyen mnémotechnique d'apprentissage d'une langue différente de celle qui lui sert de référence.

Dans cet article, nous allons voir comment un apprenant slavophone peut acquérir les modes d'expression d'une notion réputée très difficile en raison de son hétérogénéité en termes de matérialisation linguistique : l'aspect verbal. La complexité de cette catégorie du verbe nous a poussé à laisser de côté l'aspect culturel de l'apprentissage d'une langue pour nous consacrer, dans un premier temps, aux faits linguistiques en partant de la langue de référence d'un apprenant slavophone. En effet, l'incessant cassetête que cette catégorie du verbe représente dans les langues slaves par les locuteurs francophones, mais aussi par les natifs, est devenu une problématique récurrente de la linguistique théorique et descriptive, au point d'amener certains chercheurs à parler d'une toute nouvelle discipline - l'aspectologie (Guentcheva, 1990). Toutefois, dans cet article il ne s'agira pas d'étudier l'aspect en tant que catégorie propre aux verbes slaves, mais d'aller dans un sens inverse et de s'interroger sur les moyens d'apprendre l'aspect du verbe français par les apprenants slavophones, et plus particulièrement les apprenants dont la langue de référence est le macédonien.

\section{Motivations et remarques préliminaires}

Lorsque nous évoquons cette catégorie du verbe slave, la première chose qui nous vient à l'esprit est la polymorphie de sa réalisation à différents niveaux linguistiques (morphologie, syntaxe et sémantique). Le caractère unique de l'aspect a donné naissance à de nombreuses études en linguistique typologique. Dans cette lignée, nous convoquerons les travaux de B. Comrie (Comrie, 1976), O. Dahl (Dahl, 1985) et Z. Guentcheva (Guentcheva, 1990). Tous ces travaux scientifiques témoignent d'une interférence ou plutôt d'une coopération étroite entre trois catégories verbales : le temps, le mode ${ }^{1}$ et l'aspect. Nous constatons, cependant, une absence d'applications de ces travaux à la didactique des langues, ce qui permettrait de donner des mécanismes plus simples d'apprentissage de cette catégorie, aussi bien par les francophones qui apprennent d'autres langues que par les apprenants du français. Le présent travail n'a pas l'ambition d'être exhaustif et se concentrera principalement sur une des multiples facettes de l'aspect en macédonien : les préverbes. Notre but est de réfléchir à une méthode qui permettrait de mieux acquérir les modes d'expression de la catégorie de l'aspect en français, à travers une réflexion contrastive.

\section{L'aspect : une catégorie polymorphe}

Les travaux en linguistique descriptive que nous avons précités témoignent d'une diversité de la réalisation de cette catégorie du verbe sur le plan morphologique, syntaxique et sémantique. On peut observer l'aspect au niveau morphologique (en flexion ou en dérivation), ou au niveau syntaxique où l'emploi de certaines tournures ou de verbes auxiliaires change la valeur du procès. C'est pour cela qu'il convient de définir cette catégorie polymorphe et de voir comment elle se réalise en macédonien avant de s'interroger sur la manière de l'enseigner en français.

\subsection{Définitions et concepts de l'aspect}

La définition de l'aspect la plus souvent citée est celle de B. Comrie (Comrie, 1976). Comrie montre que cette catégorie est en relation étroite avec le temps et le mode de plusieurs façons : 


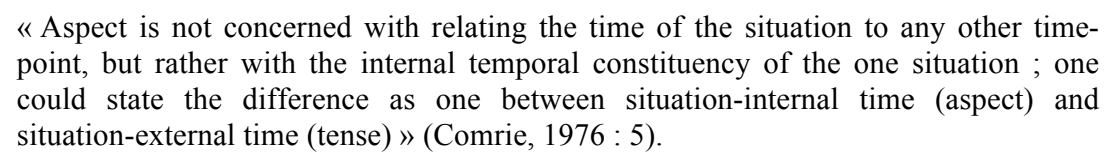

Z. Guentcheva nous explique à son tour que la «situation d'un procès dans le temps » (organisation externe) est différente de sa «situation interne ». Guentcheva met l'accent sur les «bornes» de déroulement qu'elle emprunte au précurseur de l'aspectologie russe J. Maslov (Guentcheva, 1990 : 16). En d'autres termes, la catégorie « temps » (ou « tense » en anglais) est celle qui situe le procès d'un verbe dans une «époque» (passé, présent ou futur), tandis que la catégorie de l'aspect permet de définir la nature-même d'un procès d'un point de vue organisationnel, c'est-à-dire de définir si un verbe désigne le début, la totalité, une partie ou la fin d'un procès ou, le cas échéant, un procès qui se déroule en intervalles, notamment dans le cas des verbes itératifs. Cette approche permet de situer le procès sur un axe chronogénétique par rapport à un moment $\mathrm{T}$ et de délimiter son déroulement en intervalles. De ce point de vue, le procès du verbe peut être «borné » ou «non-borné » et c'est précisément cette définition que nous allons retenir pour désigner son organisation interne, du moins pour le macédonien.

\subsection{Point de départ : l'aspect en macédonien}

Comme nous l'avons évoqué ci-dessus, l'hypothèse de la présente étude est que le point de départ d'un apprenant est la réflexion métalinguistique qu'il développe sur sa propre langue de référence qui peut être soit sa langue maternelle, soit la langue de son quotidien. Dans sa propre réflexion sur l'objet de son étude - la langue qu'il est en train d'apprendre, cette langue de référence est également une métalangue à travers laquelle l'apprenant développe une réflexion préliminaire sur ce qu'il apprend. Dans notre cas, la langue de référence - le macédonien - est une langue slave du Sud qui forme un continuum dialectal avec le bulgare. Les principales caractéristiques de cette langue à la différence des autres langues slaves sont la disparition totale du système des cas nominaux au profit d'une syntaxe entièrement analytique et la complexité du système verbal mise en évidence par de nombreux chercheurs émanant d'écoles très différentes (Friedman, 1977, Kramer, 1983, Elson, 1989, Kostov, 2013). Dans ce travail nous allons, tout d'abord donner un bref aperçu du système de la conjugaison macédonienne et, dans un second temps, voir dans quelle mesure certains éléments de la morphologie verbale peuvent s'avérer utiles dans l'enseignement du système aspectuel du français à un apprenant macédophone.

\subsubsection{Classification des verbes macédoniens}

Dans la tradition grammaticale du macédonien, il existe deux classifications des verbes : une classification formelle et une classification aspectuelle (Koneski, 1953, Kostov, 2013). La classification formelle prend en compte la forme lexicale du verbe qui est une forme dite « réalisée ${ }^{2} »:$ la troisième personne au singulier du présent de l'indicatif. Cette classification est établie en l'absence de la forme infinitive dans les langues de ce continuum slave du Sud. A l'exception du verbe sum (être), la forme lexicale de tous les autres verbes se termine par une voyelle $(a, i$ ou $e$ ), ce qui nous permet d'en distinguer trois groupes. La classification aspectuelle nous permet de distinguer les verbes imperfectifs (y compris des verbes désignant des procès itératifs) des verbes perfectifs (avec au moins une borne - initiale, finale ou initiale et finale). Il est à noter que certains verbes peuvent appartenir aux deux classes aspectuelles et c'est pour cela que nous les appelons des verbes «biaspectuels » (Kostov, 2013). Les verbes perfectifs peuvent, à leur tour, être subdivisés en sous-classes morphologiques très variées en fonction du changement de leur thème. La polymorphie de l'aspect ne peut pas être uniquement observée entre différentes langues, mais aussi au sein d'une même langue. Dans cette partie, nous allons étudier les différentes manifestations de cette catégorie en macédonien, notamment dans le cadre de la flexion et dans celui de la dérivation verbale. 


\subsubsection{Relation entre aspect et flexion verbale}

Dans la conjugaison macédonienne, nous pouvons observer une interférence entre le temps, le mode et l'aspect. Ce dernier sert, tout d'abord, d'indicateur et de filtre qui permet de déterminer à quel mode et temps on peut conjuguer un verbe suivant sa valeur aspectuelle de départ (Kostov, 2013). Ce filtrage peut être considéré comme une première règle de la conjugaison qui dans les langues du continuum dialectal bulgaro-macédonien est sensiblement plus complexe de celle du russe, tout comme le montre $\mathrm{Z}$. Guentcheva (Guentcheva, 1990 : 13). Pour simplifier cette règle, nous dirions que les verbes perfectifs peuvent réaliser pleinement leur sens au sein des formes ${ }^{3}$ perfectives, les verbes imperfectifs peuvent être employés au présent et dans la conjugaison des formes imperfectives, alors que les verbes biaspectuels peuvent être employés dans toutes les formes verbales d'un paradigme de conjugaison. La situation se complexifie dans le cadre des formes verbales périphrastiques (souvent exprimant le subjonctif ou le potentiel) dans lesquelles nous pouvons employer chaque verbe, indépendamment de sa classe aspectuelle. Ce filtrage peut être modélisé et représente un premier indice concernant la série des désinences que pouvons (et devons) employer lorsque nous voulons apprendre la conjugaison d'un verbe macédonien.

\subsubsection{L'aspect et la dérivation : les « paires aspectuelles »}

La catégorie aspectuelle d'un verbe peut être également analysée à partir de sa forme lexicale. Traditionnellement, les grammaires macédoniennes (Koneski, 1953 ou Minova-Gjurkova \& al., 1997) représentent les verbes comme mots qui peuvent prendre deux «formes» différentes: une forme imperfective et une forme perfective. Ces deux « formes »sont, en réalité, deux unités lexicales distinctes qui forment une «paire aspectuelle » d'au moins deux (ou plusieurs) verbes dont l'un est obligatoirement imperfectif et l'autre/les autres perfectif-s. Le verbe perfectif en macédonien, comme dans différentes langues slaves, est obtenu le plus souvent par l'adjonction d'un préfixe (que nous appellerons préverbe) ou d'un suffixe, appelés communément des "morphèmes de la perfectivation ». Ainsi, les verbes kaže/kažuva (fait de/ en train de dire) et iskaže (finir par/de dire) sont considérés respectivement comme la première forme (imperfective) et comme la seconde forme (perfective) d'un seul et même verbe et forment sa paire aspectuelle. Toutefois, leur sens diffère et malgré le fait que ces deux formes sont construites sur une même racine, il est plus pratique de les considérer comme deux unités lexicales distinctes et complémentaires au sein d'un paradigme: les verbes imperfectifs seront utilisés dans des formes qui exigent un verbe imperfectif et les verbes perfectifs dans des formes qui exigent un verbe perfectif. La préfixation n'est pas le seul procédé de dérivation d'un verbe perfectif à partir de son équivalent imperfectif : cette opposition lexico-aspectuelle peut être obtenue également à l'aide de la suffixation, ou plutôt du changement désinentiel : les verbes dava et dade (donner) sont également représentés comme une paire aspectuelle qui fonctionne de la même manière que l'exemple précédent. A partir de ces quelques considérations, nous pouvons constater que l'aspect en macédonien joue un rôle central dans la conjugaison. Cette complexité nous amène à limiter notre travail uniquement aux préverbes - préfixes verbaux - qui participent à la dérivation et représentent un mode très riche de création de nouvelles unités lexicales, notamment pour les verbes perfectifs.

\section{Etude des préverbes et de leurs équivalents en français}

\subsubsection{Les préverbes aspectuels en macédonien}

Dans sa Grammaire de la langue macédonienne publiée en 1953, B. Koneski dresse une liste de préverbes qui participent à la dérivation verbale. Ces préverbes sont, en réalité, des « morphèmes de dérivation aspectuelle » (Koneski, 1953 : 394). L'approche de Koneski adoptée par une grande majorité de ses contemporains, n'est pas tout à fait adaptée à tous les apprenants. L'analyse des unités du discours relève, d'une part, de la dérivation et, de l'autre, de la flexion, ce qui complique considérablement les niveaux d'analyse auxquels un apprenant est confronté dans le processus d'acquisition des règles grammaticales. Par ailleurs, les méthodes d'apprentissage du macédonien ne prennent pas suffisamment 
en compte l'origine de l'apprenant et les particularités du système linguistique. La liste de préverbes que nous allons analyser contient une quinzaine de morphèmes avec des exemples de verbes en contexte suivis de leurs valeurs sémantiques. Cependant, l'aspect en tant que concept opérationnel tel qu'il est envisagé par Comrie n'est nullement mentionné alors qu'il est évident qu'il joue un rôle prépondérant pour certains des préverbes de cette liste. Cette liste contient les préverbes suivants :

- o-, od-, ob-, obez- : Les préverbes qui commencent par [b] et par [d] appartiennent à une classe différente qui n'est plus utilisée en macédonien contemporain et c'est pour cela qu'on les précède par la voyelle [o]. Toutefois, Koneski les regroupe dans une seule classe de préverbes qui commence par $[\mathrm{o}])$.

- $\quad \mathrm{v}[\mathrm{o}[\mathrm{z} / \mathrm{s}]]-$ Ce préverbe peut avoir 3 formes différentes en fonction du phonème initial du verbe qui lui sert de base pour la perfectivation.

- do-

- za-

- iz-

- $\mathrm{na}[\mathrm{d}]-$

- po-

Avant d'étudier le sens et la valeur de certains de ces préverbes et de leurs équivalents en français, nous allons expliquer quel est leur rôle dans la dérivation verbale et, par conséquence, dans la construction des paires aspectuelles.

\subsubsection{La préfixation et la suffixation : quand est-ce que l'on construit un perfectif ?}

Les verbes imperfectifs sont considérés comme des verbes de base sur lesquels on peut effectuer des opérations de dérivation et de perfectivation. Les perfectifs, en revanche, sont des verbes à partir desquels on peut dériver des "imperfectifs secondaires » dont la plupart appartiennent à la classe des verbes itératifs. Schématiquement, les opérations de dérivation verbale peuvent être représentées de la manière suivante :

- Perfectivation préverbale : [préverbe] + [verbe imperfectif $]=[$ perfectif ' $]$

- Perfectivation à suffixation : [verbe imperfectif] + [suffixe] $=$ [perfectif ${ }^{\prime}$ ]

- Dérivation des itératifs : [perfectif' / perfectif ' $]+[$-uva] $=$ [iteratif]

Ce schéma de la dérivation justifie dans une certaine mesure le concept de paire aspectuelle que nous avons évoqué précédemment, même s'il n'est que très peu utile dans l'apprentissage de la flexion macédonienne, notamment dans le cadre de l'impératif (Elson, 1989 et Kostov, 2013). Le verbe de départ dans ce schéma de dérivation est un verbe imperfectif simple et l'emploi d'un préverbe servira à obtenir son alter-égo perfectif. Si, en revanche, on veut obtenir un verbe itératif, on emploiera un verbe perfectif sur lequel on rajoutera la désinence -uva qui est la marque de la périodicité et qui représente le mode le plus courant de création de ces verbes. Dans la terminologie actuelle, ces verbes sont caractérisés comme des « imperfectifs secondaires » sans aucune mention du terme « itératif » (Veljanovska \& al., 2007).

Le principal problème des préverbes que nous avons énumérés tient au fait qu'ils ne sont pas uniquement des préverbes liés à l'aspect, c'est-à-dire à l'organisation interne du procès d'un verbe. Leur sens peut désigner bien plus que l'organisation temporelle interne d'un procès : ils véhiculent également des informations sur l'espace et sur la quantité. De ce point de vue, le préverbe joue le rôle d'un circonstant spatial situant le procès dans un contexte beaucoup plus large que le contexte temporel. Cela complexifie son sens et il serait très inapproprié de limiter ce morphème au seul rôle aspectuel.

\subsubsection{Quel est alors le sens des préverbes ?}

L'analyse des différents préverbes de la liste de Koneski nous permet de définir deux classes distinctes en fonction de l'information qu'ils véhiculent : une classe contenant une information strictement aspectuelle propre à l'organisation interne du procès pouvant être représentée sur l'axe de la chronogénèse, et une 
autre classe qui véhicule des informations relatives non seulement aspectuelles, mais des informations liées également au contexte spatial dans lequel se déroule le procès d'un verbe donné et à la quantité du procès. Dans certains cas, l'organisation spatiale concerne plutôt le mouvement (d'un sujet ou d'un objet) à partir ou envers un endroit donné. Toutefois, il est évident que le processus de perfectivation à l'aide des préverbes est un mode productif original en macédonien et il mérite une étude contrastive avec celui $\mathrm{du}$ français qui nous permettrait d'entrevoir les différences de ces deux langues et de proposer quelques pistes d'apprentissage des modes d'expression de l'aspect en français par les apprenants macédophones. Dans un premier temps, nous allons analyser les verbes perfectifs qui véhiculent seulement des informations propres à l'organisation interne du procès pour, ensuite, nous intéresser aux verbes perfectifs construits avec des préverbes qui véhiculent d'autres informations qui ne relèvent pas uniquement de l'aspect au sens temporel.

\subsection{Etude des préverbes à dimension exclusivement aspectuelle}

Les préverbes qui véhiculent des informations liées uniquement à l'aspect d'un point de vue de l'organisation interne du procès verbal sont les préverbes $z a$ - et $d o$-. L'illustration de cette valeur strictement aspectuelle peut être faite dans l'exemple suivant :
(1) Marija
zapea
edna

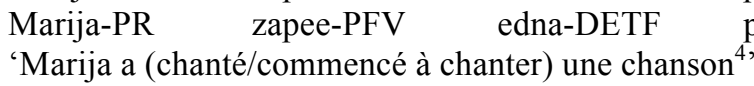
zapee-PFV
edna-DETF
pesna

Le verbe zapee - verbe perfectif - est construit sur la base du verbe imperfectif pee (chanter) auquel on ajoute le préverbe za-. Ce préverbe indique que le procès exprimé est un procès inchoatif. En effet, la seule information que le préverbe véhicule est une information sur la borne (ici initiale) et l'organisation temporelle interne et c'est pour cela que nous pouvons considérer le préverbe za- comme un préverbe exclusivement aspectuel. En français il existe deux possibilités: l'une - lexicale - se contentera d'employer la forme « chanter» dans un temps passé («Marija a chanté... / « Marija chanta... ») et l'autre - périphrastique - induit l'emploi d'un verbe semi-auxiliaire qui indique l'aspectualité inchoative (le verbe commencer : « Marija a commencé à chanter »). Lorsqu'un apprenant a affaire aux verbes qui fonctionnent de la même manière que le verbe zapee, connaissant la valeur inchoative inscrite dans l'unité lexicale, il saura, de surcroît, qu'en français il peut exploiter ces deux modes sans aucune équivoque.

D'autre part, le préverbe za- peut également exprimer des procès perfectifs avec une borne finale (des procès téliques) comme dans l'exemple suivant :
(2) Zafir
go
zamota
deteto
Zafir-PR
go-PRO
zamota- PFV
dete-NEUT
'Zafir a (couvert/fini de couvrir) l'enfant'

Le préverbe za- indique une résultativité et c'est dans ce sens qu'il a la valeur d'un préverbe qui exprime la télicité du procès. Dans le cas d'un procès non-résultatif et inchoatif, le préverbe utilisé aurait été s(smota) ce qui exprime que le fait de couvrir est au tout début de son déroulement. Dans ce cas comme dans le précédent, le sens télique en français sera obtenu par l'emploi d'un temps perfectif (passé composé ou passé simple) ou, le cas échéant, à l'aide d'un circonstant temporel qui donne une précision sur la nature télique du procès - un verbe semi-auxiliaire («finir de/par» ou, éventuellement, « venir de $\gg$ ).

Le préverbe do-, quant à lui, exprime uniquement des procès en train de se terminer et de se compléter. Sa valeur peut être illustrée avec l'exemple suivant :
(3) $\mathrm{Ja}$
dotera
salata
ja-PRO
dotera- PFV sala-F
'Il/elle a (décoré/fini de décorer) la salle' 
En français, le sens équivalent de ce préverbe peut être obtenu en employant une périphrase verbale avec le semi-auxiliaire «finir de + infinitif » d'un procès antérieur inachevé, voire suspendu, et qui nécessite d'être repris pour mener à terme son accomplissement.

Dans les deux cas, nous supposons que l'apprenant connaît la valeur sémantique du préverbe ce qui pourrait lui servir d'indice de choix des unités avec lequel il va obtenir le sens équivalent en français, ce qui facilite considérablement le processus d'apprentissage. Ce processus ne passe pas uniquement par la simple traduction des faits, mais aussi par une transposition réflexive sur les mécanismes de la langue de référence et de la langue d'apprentissage.

\subsection{Etude des préverbes à dimension spatio-temporelle et quantitative}

Mis à part la valeur aspectuelle du verbe, ces préverbes véhiculent également une information relative à l'espace. Enfin, ils peuvent également quantifier le procès d'un verbe. Tel est le cas, par exemple, de is/iz- qui est un préverbe qui véhicule une information sur le lieu vers lequel ou à partir duquel se déroule un procès qu, dans les deux cas, est un procès avec une borne temporelle finale :

$\begin{array}{llll}\text { (4) Ja } & \text { izvadi } & \text { kolata od } & \text { garažata } \\ \text { ja-PRO } & \text { izvadi- PFV } & \text { kola-F od-PREP } & \text { garaža-F } \\ \text { 'Il/elle a (sorti/fini de sortir) l'auto du parking' } & \end{array}$

Le préverbe iz- se réfère ici à un endroit duquel on extrait quelque chose. Le complément kolata est suivi d'un complément (od garažata). De ce fait, le préverbe indique la nature ablative du procès du verbe ce qui en français peut s'exprimer par le complément circonstanciel de lieu introduit par la préposition « de » ou «depuis ». Dans certains cas, le sens de ce préverbe peut renforcer également un procès inessif, surtout lorsque le verbe indique un mouvement vers ou dans un endroit précis. Dans ce cas, c'est la préposition so qui introduit le complément du COD comme dans l'exemple suivant :
(5) Ja
ispolni
kolata so
knigi
ja-PRO
ispolni- PFV
kola-F so-PREP
kniga-FPL

'Il/elle a (rempli/fini de remplir) l'auto de livres'

Le COD suivi du complément de nom so knigi indique aussi qu'il s'agit d'un lieu dans lequel on apporte une certaine quantité de quelque chose. Ce procès désigne un mouvement vers un lieu (kolata) ce qui nous amène à qualifier ce préverbe de préverbe véhiculant une information sur la quantité et sur la direction, et pas uniquement sur l'aspect perfectif du verbe et sa borne finale. Le même fonctionnement peut être analysé dans les verbes perfectifs construits avec le préverbe na[d]- qui véhicule, cette fois-ci, une information quantitative qui surpasse un procès auquel on le compare, même de façon implicite.
(6) Go
go-PRO
nadminav Vardar.
nadmine-PFV Vardar-PR
'(J'ai surpassé/Je suis passé devant/J'ai joué mieux que) Vardar'

Dans cet exemple, nous avons clairement une comparaison d'un procès avec un état ou un procès antérieur. Le procès désigné est quantitativement ou qualitativement supérieur à celui auquel on le compare et dont le sujet était le COD (ici Vardar). Concernant l'aspect au sens de l'organisation interne du procès, il s'agit d'un perfectif pur et simple. Dans ce cas, contrairement aux autres préverbe, le préfixe sur- en français joue le rôle équivalent dans la majorité des cas. Toutefois, avec certains verbes (ex. jouer), il ne fonctionne pas car il dénote une exagération et non pas une quantification ou qualification positive du procès comme c'est le cas en macédonien. De ce fait, l'apprenant peut faire appel à d'autres moyens d'expression de la quantité comme les locutions adverbiales de manière (ex. mieux que, plus que etc.) qui n'induiraient pas l'emploi d'un faux ami.

Enfin, le préverbe po- est un préverbe grâce auquel on construit un procès borné des deux côtés de l'axe de la chronogénèse. Toutefois, ce procès est quantifiable dans le temps et dans la quantité de l'effort fourni par le sujet qui est une quantité délimité, voire faible. Nous pouvons illustrer ces valeurs à travers l'exemple suivant : 


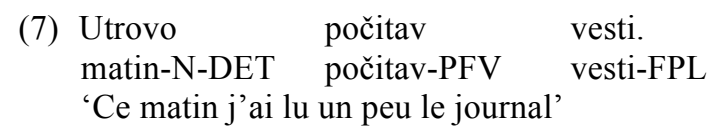

En macédonien, la quantification du procès perfectif est entièrement contenue dans le préverbe po- et il dénote indéniablement un procès borné avec un début et une fin sur l'axe de la chronogénèse. En outre, ce préverbe donne des informations essentielles sur la quantité temporelle du procès. Un équivalent possible en français serait l'emploi de certains verbes qui se terminent par la désinence -oter qui exprime la diminution d'un procès, mais aussi l'emploi des adverbes de quantité (beaucoup, peu, pendant $\mathrm{x}$ temps etc.).

\subsection{L’aspect : une catégorie indéniablement polymorphe}

Dans ce travail qui est loin d'être exhaustif, nous avons essayé de dresser une analyse sémantique, syntaxique et morphologique de l'expression de l'aspect en macédonien, et nous avons cherché ses marqueurs équivalents en français afin d'établir des passerelles entre la langue de référence et la langue d'apprentissage. Ce que cette modeste analyse nous apprend c'est que l'aspect est une catégorie analysable aussi bien sur le plan morphologique que sur le plan syntaxique et sémantique et il est difficile de l'insérer dans des cases rigides opérationnelles dans l'apprentissage d'une langue dans un sens comme dans l'autre. Toutefois, ce que nous pouvons apprendre des préverbes en macédonien c'est qu'ils sont difficilement transposables avec des moyens équivalents en français et qu'ils véhiculent d'autres informations que l'organisation interne temporelle du procès. De ce point de vue, ce qui serait intéressant c'est de réfléchir à la relation entre deixis et aspect verbal, non seulement en macédonien et en français qui est la paire linguistique étudiée dans cet article, mais à d'autres paires de langues dans une perspective didactique. Dans tous les cas, concernant les préverbes, nous pouvons représenter les équivalences en macédonien et en français à travers le tableau suivant :

\begin{tabular}{|c|c|c|}
\hline Préverbe & Valeur sémantique & Marqueurs aspectuels en français \\
\hline za- & $\begin{array}{ll}\text { - } & \text { procès inchoatif } \\
\text { - } & \text { procès télique }\end{array}$ & $\begin{array}{l}\text { - passé composé, passé simple ou autre } \\
\text { temps exprimant un procès perfectif; } \\
\text { - forme verbale périphrastique } \\
\text { indiquant le début ou la fin du procès; }\end{array}$ \\
\hline do- & $\begin{array}{l}\text { - } \quad \text { procès télique } \\
\text { - } \quad \text { procès inessif ou allatif }\end{array}$ & $\begin{array}{l}\text { - passé composé, passé simple ou autre } \\
\text { temps exprimant un procès perfectif; } \\
\text { - } \quad \text { forme verbale périphrastique } \\
\text { indiquant le début ou la fin du procès ; } \\
\text { - compléments circonstanciels de lieu à } \\
\text { valeur inessive ou allative; }\end{array}$ \\
\hline iz-/is- & $\begin{array}{l}\text { - } \quad \text { procès élatif ou ablatif } \\
\text { - } \quad \text { procès télique }\end{array}$ & $\begin{array}{l}\text { - la valeur élative ou ablative peut être } \\
\text { exprimée à travers des compléments } \\
\text { circonstanciels de lieu ; } \\
\text { - certains adverbes (ex. entièrement) } \\
\text { peuvent également constituer un } \\
\text { élément de renforcement de la télicité } \\
\text { et de l'accomplissement du procès } \\
\text { d'un verbe; }\end{array}$ \\
\hline nad- & - $\quad$ procès télique & $\begin{array}{l}\text { - verbe équivalent (si possible construit } \\
\text { avec le préfixe « sur- } » \text { ou « dé- } »)\end{array}$ \\
\hline
\end{tabular}




\begin{tabular}{|c|c|c|}
\hline & $\begin{array}{l}\text { - } \text { sublatif; } \\
\text { - } \quad \text { comparaison avec un } \\
\text { procès antérieur; }\end{array}$ & $\begin{array}{l}\text { - adverbes de quantité introduisant des } \\
\text { compléments circonstanciels de } \\
\text { manière (plus que, mieux que etc.); } \\
\text { - passé composé, passé simple ou autre } \\
\text { temps exprimant un procès perfectif; }\end{array}$ \\
\hline po- & $\begin{array}{l}\text { - } \begin{array}{l}\text { procès délimité entre } \\
\text { deux bornes; }\end{array} \\
\text { - itération (avec les } \\
\text { imperfectifs } \\
\text { secondaires); }\end{array}$ & $\begin{array}{l}\text { - adverbes de quantité introduisant des } \\
\text { sur la durée ou sur la qualité d'un } \\
\text { procès (un peu, beaucoup, x heures } \\
\text { etc.); } \\
\text { - des verbes indiquant une durée } \\
\text { spécifique (pianotter, tapoter) } \\
\text { accompagnés, le plus souvent, d'un } \\
\text { complément circonstanciel de temps; }\end{array}$ \\
\hline
\end{tabular}

Tableau 1 : Certains préverbes macédoniens et leurs équivalents en français ${ }^{5}$.

\section{Conclusion et perspectives de la présente étude}

La typologie des aspects verbaux en macédonien est clairement établie dans différentes études en linguistique théorique et descriptive. Si jusqu'à maintenant nous avons eu l'habitude de voir des méthodes qui proposent un enseignement plus facile de l'aspect du verbe des langues slaves, notre étude a voulu aller dans l'autre sens et de voir comment les apprenants slavophones, et plus particulièrement les apprenants du macédonien, peuvent exploiter les connaissances de leur propre système linguistique de références et de voir ce qu'ils peuvent utiliser en français dans un processus non seulement d'apprentissage, mais aussi de traduction depuis cette langue.

D'autre part, à la lumière de différents travaux, et notamment de ceux de J.-J. Franckel (Franckel, 1989) et de V. Friedman (Friedman, 1977), nous pouvons constater une grande hétérogénéité des marqueurs aspectuels dans les deux langues. Une étude contrastive détaillée nous permettrait d'établir des comparaisons plus fines qui pourraient être utilisées dans le processus d'apprentissage de l'aspect en français standard pour les besoins des apprenants des langues slaves en général, et notamment pour ceux dont les langues slaves du Sud représentent des langues de référence dans lesquelles ils ont développé un minimum de réflexion métalinguistique. Toutefois, ce processus n'est pas simple car ces connaissances métalinguistiques sont le résultat d'une scolarisation - paramètre essentiel qui nécessite une prise en compte dès le début de l'apprentissage d'une langue étrangère.

Ayant eu la possibilité d'observer et de pratiquer l'apprentissage des langues slaves et du français in situ, nous avons toutes les raisons de croire qu'un enseignant qui dispose d'un minimum de connaissances du système linguistique de référence de l'apprenant peut considérablement remonter l'autoestime de celui-ci et à le valoriser dans ce processus d'interaction réflexive. Expliquer une langue en la comparant à une autre n'est autre qu'une réflexion introspective qui vise à déchiffrer les moyens d'expression de certains phénomènes linguistiques dans la langue de référence, avant de chercher (et de trouver) ses équivalents dans la langue que l'on veut (faire) apprendre. C'est dans ce sens que nous définirions l'apprentissage comme un processus avant tout introspectif et réflexif renvoyant constamment au sujet-apprenant et rendant l'enseignement des langues plus inclusif, aussi bien pour les langues que pour les apprenants. Par ailleurs, et dans une perspective de modélisation informatique de la flexion, une étude des différentes classes aspectuelles nous permettrait d'améliorer considérablement les systèmes existants de flexion automatique des verbes, fondés sur les propriétés flexionnelles de cette classe incontestablement complexe d'une langue à une autre. 


\section{Références bibliographiques}

Arrivé, M. (1986), La Grammaire d'aujourd'hui, Paris : Flammarion.

Blevins J. (2013), Word-based morphology from Aristote to modern WP, The Oxford handbook of the history of linguistics 16, Oxford : Oxford University Press, pp. 375-396.

Bonnard H. (1981), Code du français courant, Paris : Magnard.

Cassin B. (2004), Vocabulaire européen des philosophies, Paris : Seuil - Le Robert.

Comrie, B. (1976). Aspect. An introduction to the study of verbal aspect and related problems. Cambridge : Cambridge University Press.

Comrie, B. (1989). Perfectif et télique, Travaux de linguistique, 19, pp. 57-66.

Confais J.-P. (1990), Temps, mode, aspect : les approches des morphèmes et leurs problèmes à l'exemple du français et de l'allemand, Collection Interlangues, Toulouse : Presses universitaires du Mirail.

Dahl, O. (1985), Tense and aspect systems, Oxford : Basil Blackwell Ltd.

Elson, M.-J. (1989), Macedonian verbal morphology : a structural analysis, Columbus-Ohio : Slavica.

Franckel J.-J. (1989), Etude de quelques marqueurs aspectuels du français, Genève-Paris : Librairie Droz.

Friedman V. (1977), The grammatical categories of the macedonian indicative, Columbus : Slavica.

Friedman V. (1989), Upotrebata na glagolskite vreminja vo makedonskite i vo drugi balkanski poslovični izrazi, Makedonski jazik 40-41, Skopje : Institut za makedonski jazik « Krste Misirkov ».

Foulon-Hristova J. (1997), Les modes de narration en macédonien, Collection Linguistique, Langues \& Ecritures, Paris : L'Asiathèque.

Foulon-Hristova J. (1998), Grammaire pratique du macédonien, Collection Langues \& Mondes, Paris: L'Asiathèque.

Galton H. (1986), Markedness in macedonian verbal morphology, Prilozi, XI 2 -Oddelenie za lingvistika $i$ literaturna nauka, Skopje : Makedonska akademija na naukite i umetnostite, pp. 77-82.

Gosselin, L. (2005). Temporalité et modalité. Bruxelles : Duculot.

Grevisse, M. \& Goosse, A. (2011), Le bon usage - Grammaire, langue française, Paris-Louvain-la-Neuve : De Boeck - Duculot.

Guentchéva, Z. (1990). Temps et aspect: l'exemple du bulgare littéraire contemporain, Collection Sciences du Langage, Paris: Presses du CNRS.

Guentchéva, Z \& Desclés J.-P. (2010), Les référents aspecto-temporels : une approche formelle appliquée au français,

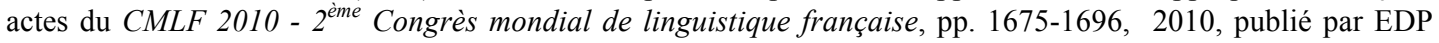
Sciences (www.linguistiquefrancaise.org), [DOI : http://dx.doi.org/10.1051/cmlf/2010259].

Hogeveg L. \& al. (éd.) (2009), Cross-linguistic semantics of tense, aspect and modality, Collection Linguistik aktuell, Amsterdam-Philadelphia : J. Benjamins.

Jakobson R. (1963), Essais de linguistique générale 1, Paris : Editions de Minuit.

Kepeski, K. (1946), Makedonska gramatika, Skopje : Državno knigoizdatelstvo na Narodna Republika Makedonija.

Koneski, B. (1953), Gramatika na makedonskiot literaturen jazik, Skopje : Prosvetno delo.

Kostov J. (2013), Le verbe macédonien : pour un traitement informatique de nature linguistique et applications didactiques (réalisation d'un conjugueur), thèse de doctorat soutenue le 7 décembre 2013, INALCO, Paris.

Kramer C. (1986), Analytic modality in macedonian, Munich : Verlag Otto Sagner.

Lunt, H. (1952), A Grammar of the macedonian literary language, Skopje : Državno knigoizdatelstvo na Narodna Republika Makedonija. 


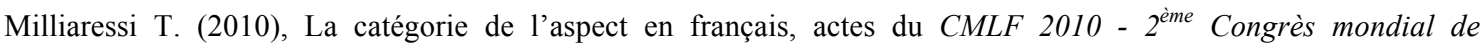
linguistique française, pp. 1399-1413, 2010, publié par EDP Sciences (www.linguistiquefrancaise.org), [DOI : http://dx.doi.org/10.1051/cmlf/2010197].

Minova-Gjurkova L. \& al. (1997), Gramatika na makedonskiot jazik za srednoto obrazovanie. Skopje : Prosvetno delo.

Minova-Gjurkova L. (2006), Gramatika na makedonskiot standarden jazik za stranci, Štip : 2-ri avgust.

Minova-Gjurkova L. \& al. (2008), Opšta gramatika na makedonskiot jazik. Skopje : Prosvetno delo.

Mišeska-Tomič O. (2012), A grammar of macedonian, Bloomington : Slavica.

Novakova I. (1995), Sur les systèmes aspecto-temporels du français et du bulgare, Contrastive linguistics 6/1995, Sofia : Université de Sofia, pp. 5-14.

Novakova I. (2001), Fonctionnement comparé de l'aspect verbal en français et en bulgare, Revue des études slaves LXXIII/1, pp. 7-23.

Riegel M. \& al. (2009), Grammaire méthodique du français ( $7^{\text {ème }}$ édition revue et augmentée), Paris: Presses Universitaires de France.

Schönn D. A. (1984), The Reflective Practitioner: How Professionals Think in Action, New York : Basic Books.

Tofoska S. (1997), Izveduvanje na telični glagoli od osnovi od kontinuativen vid, Godišen zbornik na Filološkiot fakultet, Skopje : Univerzitet Sv. Kiril i Metodij, pp. 325-347.

Tofoska S. (1998), Mehanizmi na generiranje telična konfiguracija vo makedonskiot jazik, Semantika i struktura na slovenskiot vid III, Skopje : Univerzitet Sv. Kiril i Metodij.

Vandeloise C. (1986), L'espace en frnaçais: sémantique des prépositions spatiales, Collection Travaux linguistiques, Paris : Editions du Seuil.

Veljanovska K. \& al (2007), Pregled na vidskite formi na počestite glagoli vo makedonskiot jazik. Skopje: Univerzitet Sv. Kiril i Metodij.

\footnotetext{
${ }^{1}$ Nous distinguerons la catégorie du «mode» (verbal)» de la notion de «modalité » qui, à notre sens, a toute sa légitimité dans l'étude des unités linguistiques dans une perspective énonciative.

${ }^{2}$ Nous empruntons ce terme au linguiste britannique J. Blevins (Blevins, 2013).

3 Pour des raisons pratiques, nous préférons le terme «forme verbale» aux termes «temps verbal» et «mode verbal ». En effet, dans certaines formes, la catégorie de temps n'est pas opérationnelle. Tel est le cas, par exemple, du gérondif macédonien qui exprime un procès duratif et se construit exclusivement avec des verbes imperfectifs.

${ }^{4}$ Pour des raisons de lisibilité, nous avons présenté nos exemples selon la norme Leipzig Glossing Rules disponible sur Wikipedia (https://en.wikipedia.org/wiki/List_of_glossing_abbreviations, dernière consultation le 10 décembre 2015).

${ }^{5}$ Ce tableau présente une première approximation de cinq préverbes de la liste de Koneski que nous avons présentés dans les exemples analysés.
} 